\title{
More than a Few LAB Alleviate Common Allergies: Impact of Paraprobiotics in Comparison to Probiotical Live Cells
}

\author{
Nditange Shigwedha ${ }^{1,2,3 *}$, Lanwei Zhang ${ }^{*}$, Liubov Sichel ${ }^{3 *}$, Li Jia ${ }^{4 *}$, Pimin Gong1, Wenli Liu ${ }^{1}$, \\ Shumei Wang1, Shuang Zhang1, Xue Han'1, Wei Gao' \\ ${ }^{1}$ School of Food Science and Engineering, Harbin Institute of Technology, Harbin, China \\ ${ }^{2}$ Department of Food Science and Technology, University of Namibia, Windhoek, Namibia \\ ${ }^{3}$ Pure Research Products LLC, Boulder, USA \\ ${ }^{4}$ Wyeth Nutrition, Suzhou, China \\ Email: ${ }^{*}$ nditange@gmail.com, ${ }^{*}$ zhanglw@hit.edu.cn, ${ }^{*}$ Isichel1@gmail.com, ${ }^{*}$ jialicad@gmail.com
}

Received March 2014

\section{Abstract}

The evidence in this paper indicates that the alarming increase of common allergies can be reduced by the intake of particular "probiotics" or "paraprobiotics" along with food. This appears to build a consensus in the pharmaceutical and food communities about the role of probiotics in the prevention and treatments of common allergies. Food allergy is one of the common allergies, defined as an adverse health effect arising from a specific immune response that occurs reproducibly on exposure to a given food. Improving the digestion of foods and maintaining a healthy gastrointestinal (GI) tract is certainly critical to controlling food allergens. Therefore, the association between a leaky gut or an impaired intestinal permeability and food-allergenic reactions is explained. Gluten has been found to be somehow a justification for protein allergy, and should, therefore, be avoided by people with celiac disease. In several, in vitro models, surface layer (Slayer) proteins of selective paraprobiotics have shown potential in alleviating food allergies and intestinal disorders. Notably, lactobacilli paraprobiotics have proven to be the immediate immunomodulators against common allergies and other diseases, including viral (flu, hepatitis $\mathrm{C}$ ), bacterial (bronchitis), asthma, chronic fatigue, fibromyalgia, gastrointestinal distress, and autism disorders in humans.

\section{Keywords}

Paraprobiotics, Food Allergy, S-Layer Proteins, Immunoglobulin E (IgE), Probiotical Cell Lysates, Lactic Acid Bacteria (LAB)

\section{Introduction}

Probiotics are live microorganisms that when administered in adequate amounts, confer a health benefit on the

${ }^{*}$ Corresponding authors.

How to cite this paper: Shigwedha, N., et al. (2014) More than a Few LAB Alleviate Common Allergies: Impact of Paraprobiotics in Comparison to Probiotical Live Cells. Journal of Biosciences and Medicines, 2, 56-64.

http://dx.doi.org/10.4236/jbm.2014.23008 
host [1]. In contrast, paraprobiotics are non-viable microbial cells (intact or broken) or crude cell extracts which when administered (orally or topically) in adequate amounts, confer a benefit on the human or animal consumer [2]. Probiotics, paraprobiotics, and their composites have been confirmed to confer health benefits to the hosts depending on the strain and ingested amount [3]. These include the suppression of the inflammatory disorders, inflammatory bowel disease (IBD), rheumatoid arthritis, atopic dermatitis, establishment of tolerogenic immune response, and more. The anti-inflammatory activities and modulation of local and systemic immune response by paraprobiotics are becoming extremely popular in these days. Various species of lactic acid bacteria (LAB), Bacillus, and fungi such as Aspergillus and Saccharomyces have been used over the years in the pharmaceutical and food industries. However, a few have gained probiotic status. Most of these belongs to the genera Lactobacillus, Streptococcus, Bifidobacterium, and Lactococcus. Numerous probiotics have gained recognition for their therapeutic health benefits, including help to regulate digestion, aid in lactose digestion, resistance to enteric pathogens (Salmonella sp., Escherichia sp., Listeria monocytogenes, Clostridium sp., and Candida sp.), the anticolon cancer strength, immune system modulation, anti-allergy, and hepatic and anti-encephalopathy, as well as for having a protective effect against diarrhea. Both food and pharmaceutical industries have a well-established market in the use of strain-specific probiotics and/or paraprobiotics for the positive health benefits on humans. They are typically found in beverages or designed as dietary adjuncts (in tablet or capsule formats). They can also be integrated into baby foods or in different milk powder formulations.

Food allergy, which all the main emphasis of this review, is an abnormal immune response of a person prompted by the consumption of certain food proteins [4]. The incident of allergic reactions to foods is one of the most challenging global problem because of its increasing prevalence. An estimate made by the World Allergy Organization indicates that about 220 - 250 million individuals around the world are suffering from food allergies to the range of approximately 5\% - 8\% (in infants and children) and 1\% - 2\% (in adults) [4]. The reason the prevalence of food allergies continues to grow has been worrisome for many years. The hygiene hypothesis proposes that the intake of nearly sterile foods and improved sanitation have increased and changed our exposure to environmental microbes. In addition, the use of antimicrobial medications or vaccinations might have contributed to the development of allergic reactions to certain foods in human [5]. Until now, there is no singular medication for common allergies and the solution appears to reside within probiotics and their related products. Many people believe that the adverse immune reactions can only be prevented by circumventing the offending allergens. However, avoidance of the offending allergens is difficult because trace amounts of allergens in food can trigger an allergic reaction that may include fatal anaphylaxis. Cow milk, eggs, soy, wheat, peanuts, tree nuts, fish, and crustacean (shellfish) cause most of the food allergic reactions, and some are also the first foods causing anaphylaxis. Food served in restaurants or other food service operations may be contaminated with undeclared allergens. The undeclared allergens may accidentally come in processed foods through various ways, such as improper handling and labeling, cross-contact (in-process and post-process), and ineffective or insufficient equipment sanitation procedures.

\section{Background of a Healthy Digestive Tract and Food Allergy}

Food allergy is an immunological phenomenon. Reasons for both food allergies and non-allergic food hypersensitivity (formerly known as food intolerance) is a "leaky gut” or increased intestinal permeability (Figure 1) [6]. When the gut lining is damaged by either pathogenic bacteria, poor diet, sulfites, alcohol, smoking, gastrointestinal (GI) infection, inflammations, or stress, the partially digested food gets into the bloodstream (Figure 1) and can cause an immune response. In a healthy digestive system (Figure 1(A)), the nutrients in food are digested exclusively into their smaller units (for example, glucose, fructose, and galactose for carbohydrates; and amino acids for protein) before being absorbed into the bloodstream. The tight junctions of the intestinal lining act like closed doors that prevent allergens, incompletely digested-food constituents, bacteria, and toxins from passing into the bloodstream. With a leaky gut (Figure 1(B)), the tight junctions do not function as they should. The incompletely digested-food particles, toxins, allergens, and bacteria in the intestines can go right into the bloodstream. These compounds can overwhelm the immune system, trigger food sensitivities, and affect the functioning of the body (causing or worsening joint pain, skin problems, allergic symptoms or autoimmune conditions, headaches, and so on [6].

It is necessary to show that food allergy is often associated with hypochlorhydria or less stomach acid [7]. Reference [8] reported that the food proteins that are ingested and appropriately digested are harmless. Therefore, understanding the basic knowledge of digestion is necessary. Digestion is dependent on a 3-stage process 


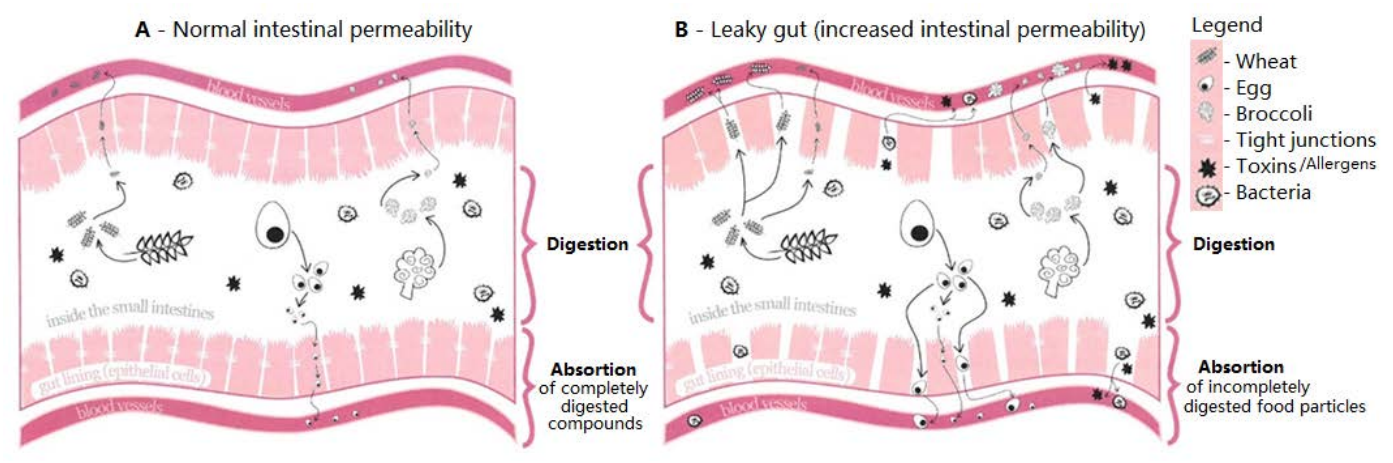

Figure 1. Normal intestinal permeability (A), Leaky gut or increased intestinal permeability (B). Modified from reference [6].

of breaking down foods. The $1^{\text {st }}$ stage, called predigestion, occurs in the mouth and esophagus. The $2^{\text {nd }}$ stage takes place in the stomach and relies on stomach acid and the enzyme pepsin. The $3^{\text {rd }}$ stage occurs in the small intestine, where additional digestive enzymes complete the breakdown process, and nutrient absorption finally occurs. Predigestion of food in the mouth and esophagus can be compromised by the simple act of failing to chew the food thoroughly. Moreover, it can be influenced by the food's people choose to eat. Foods that are low in enzyme contents or that are highly processed can lead to poor predigestion, when entering the stomach. Over time, this takes a toll on how much stomach acid human body can produce. Aging also effectively reduces the amount of stomach acid that humans could produce. Therefore, aging coupled with a reduction in stomach acid (triggered by poorly predigested food passing into the stomach), can affect the $2^{\text {nd }}$ stage of digestion. The $3^{\text {rd }}$ stage of digestion can be affected by a deficiency of pancreatic digestive enzymes, leading to a compromised digestion of food particles [7]. Apart from salivary and pancreatic $\alpha$-amylases that are only needed in starch digestion, humans do not have the intestinal enzymes needed to digest dietary fibers. Consequently, they make their way intact to the colon, where they will be bacterially fermented, together with unabsorbed nutrients.

Compromised digestion, no matter the cause, only means that food allergens are entering the bloodstream. Proteins in the bloodstream lead to immune response and food allergy [6] [7]. Another outcome of hypochlorhydria is fermentation of undigested food in the bowel, which results to symptoms such as gas, bloating, and upset feeling. This is not happening when consuming properly digested and fermented foods, preferably with the presence of active probiotics.

\section{Fermented Foods in Combating Common Allergies and Food Intolerances}

Probiotic bacteria in fermented foods can be fundamental constituents to help rebalance the gut flora. It is documented that various probiotics can detoxify the body, synthesize essential nutrients, and boost the immune system [6]. The popular fermented foods with recognized probiotics include yogurt, sour cream, kefir, sauerkraut, kimchi, crème fraîches, kombucha, and more. Sauerkraut and pickles sitting on the shelf are less likely to contain live probiotics in them. Here, the recommended advice is to look for fermented foods that are refrigerated.

Dietary studies have advocated that long-term consumption of yogurt can reduce some of the clinical symptoms of allergy in adults with atopic rhinitis or nasal allergies, and can lower serum levels of Immunoglobulin E (IgE), particularly among the elderly [9]. Besides, milk whey formula supplemented with L. rhamnosus GG could alleviate some aspects of atopic allergy and intestinal inflammations among infants [9]. In breast-fed infants whose mothers consumed L. rhamnosus GG, the clinical scores of eczema were reduced excessively [10]. The mode by which common allergies are reduced with the consumption of fermented foods is somewhat strange, but several theories exist, including the ability of probiotics to hydrolyze allergenic food molecules enzymatically, the stabilization of the gut mucosa sufficiently, and systemically reduce the uptake of food-borne allergens [9]. A distraction of the intestinal microflora (mainly endogenous probiotic bacteria) has been reported as one of the significant events related to allergic diseases [11].

Bifidobacteria and lactobacilli are the main yogurt strains, and their role in allergic and healthy infants have been intensively investigated. The 2 groups have been shown to modulate the host's immune responses to po- 
tentially damaging antigens, and down-regulate hypersensitivity reactions in humans [11] [12]. Probiotics have been also shown to modulate the phagocytosis differently in allergic and healthy individuals. In allergic persons, down-regulation of the inflammatory response was detected, whereas, in healthy individuals, there was an immunostimulatory effect [12] [13]. Other probiotic strains of the Bacillus genus are also reported to aid in digestion while at the same time reduces allergenicity [14]. Catalase and subtilisin produced by Bacillus species have been shown in vitro somehow, to stimulate Lactobacillus sp. growth in a synergistic way [15]. However, failure to reduce the risk of atopic dermatitis by L. acidophilus supplementation has been reported [3] [16].

Lactose-intolerant people are advised to ingest daily fermented dairy products (yogurt and/or kefir) because living probiotics in those products would have enough time to ferment all the lactose as opposed to only one portion of various yogurts. In addition, it is advisable for allergic/intolerant people to casein (the main protein found in milk), or to the main whey proteins ( $\alpha$-lactaglobulin and $\beta$-lactoglobulin found in milk of ruminant species), to avoid fermented dairy products. For the probiotic strains in fermented products ( $\mathrm{pH}$ around 4.5) to reach their benefits, these food products should not be heated and should be kept cold or refrigerated at $4{ }^{\circ} \mathrm{C}$ before consumption. At this temperature, the survival counts (log CFU/mL) are expected to last much longer during storage than the products stored at the room temperature.

\section{Paraprobiotics in Combating Common Allergies and Additional Diseases}

Research has demonstrated that paraprobiotics too subscribe to the overall health status of humans. The probiotical cell fragments (PCFs), currently enjoying growing popularity as immunomodulators contains, among other components, cell wall peptidoglycans and DNA fragments immensely contribute to the health status of humans. The paraprobiotic product “Del-Immune $\mathrm{V}^{\circledR}$,, is manufactured by Pure Research Products, LLC, Boulder, Colorado, USA, as a food supplement for immediate immune system support [17]. CytoFlora ${ }^{\circledR}$ is another paraprobiotic product containing cell walls of L. acidophilus, L. plantarum, L. rhamnosus, L. bulgaricus, L. salivarius, L. casei, L. reuteri, L. sporogenes, B. bifidum, B. infantis, B. longum, and S. thermophilus [18].

Preliminary experimental and clinical data indicated that the cell lysates of $L$. rhamnosus $\mathrm{V}$ are highly effective in prevention and treatment of infectious diseases, common allergies (food allergy, bronchitis, hay fever, and asthma), hepatitis C, chronic fatigue, and fibromyalgia [17]. The PCFs of L. rhamnosus V lysates are technically validated to: accelerate normalization of humoral immunity; to reinforce the actions of natural killer (NK) cells and phagocytosis; to increase production of interleukins (IL)-1, IL-2, and tumor necrosis factors (TNFs); significantly increase T-lymphocyte cells; to normalize the amount of IgAs and IgGs; to increase resistance against bacterial and viral infections; to prevent side effects against antibiotics and cyclophosphamide usage; to induce cytosuppression, and significantly reduce toxic side effects [17]. These paraprobiotics motifs have a combined effect on colon cancer and useful in making chemo- and radiotherapy possible without doubt [17] [19], and are strongly involved in the regulation of T helpers (Th1 or Th2 paths) of the immune responses. As part of PCFs, peptidoglycans (muramyl peptides; MPs, muramyl dipeptides; MDPs, muramyl tripeptides; MTPs), lipoteichoic acids (LTAs), and DNA motifs are suggested to be promising LAB ingredients for healthcare products. These have been shown to activate the neutrophils through nucleotide-binding oligomerization domain-containing protein 1 (NOD-1) and NOD-2. The neutrophils are the main cellular component of the innate immunity system.

Another dietary supplement Delpro ${ }^{\circledR}$ [20], consisting of a series of 5 probiotic strains (L. acidophilus, L. casei, L. delbrueckii, B. longum, B. bifidum; 2 billion CFUs each) formulated with the immunomodulator Del-Immune $\mathrm{V}^{\circledR}$ (L. rhamnosus V lysate; $8 \mathrm{mg}$ ) has indicated significant advantage in the treatment of GI pain and autism spectrum disorder (ASD) among children [20]. Paraprobiotics may also be able to provide health benefits despite a brief exposure to stomach acidity and bile salts following ingestion. Figure 2 illustrates that, although a log-scale reduction in probiotic cell viability may occur during digestion, a number of probiotics may still pass through the gut, depending on the dose [21]. Some cells may be injured or die, but through their PCFs, they too would be able to provide the beneficial effects on human health. Therefore, PCFs as paraprobiotic fragments may serve as "novel nutraceutical ingredients" and this establishes a consensus in the food and medical industries about their role in the development of a strong immune system, anti-allergy, anti-inflammation abilities, and prevention and treatments of various infections or diseases in humans and animals.

\section{Probiotic and Paraprobiotic Formulations for Maintaining Health Status}

To what seems to be effective, it is advisable that both children, adults, and senior citizens should accompany 


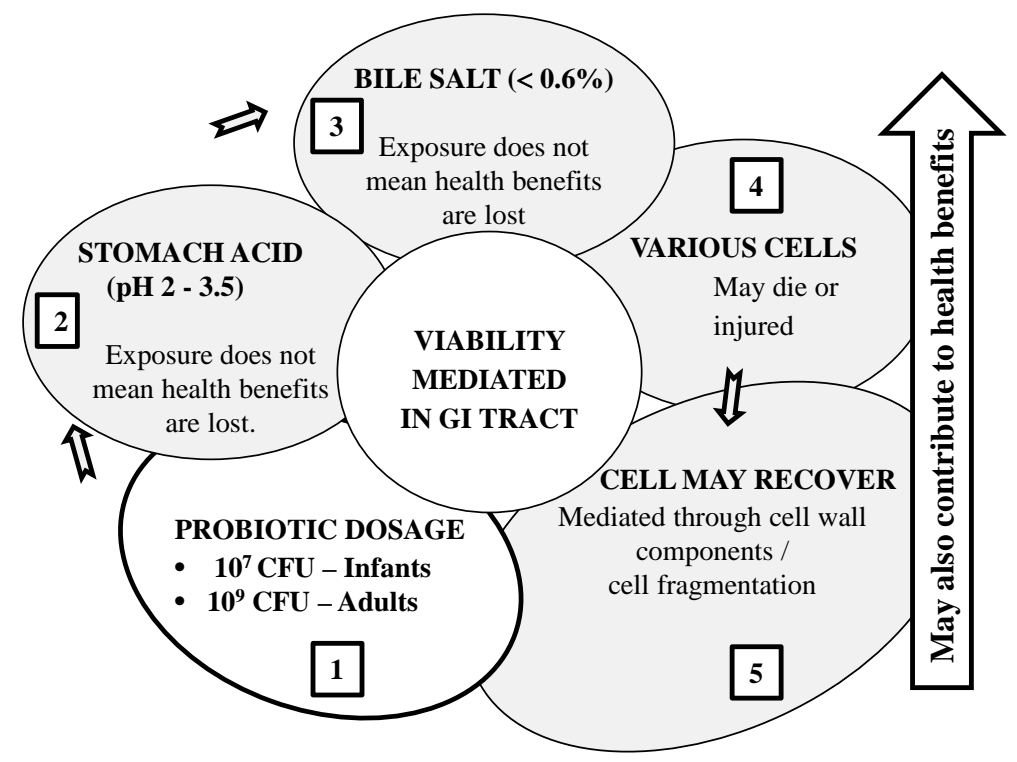

Figure 2. The 5 steps of how probiotic bacteria may recover from excessive exposure of stomach acidic and high bile salts.

their diets with probiotics cultures and/or PCFs on a daily basis. The fermented foods, probiotic dietary supplements, and paraprobiotic formulations offer a combination of different probiotic strains or their valuable cell fragments which exert the beneficial effects to the host. Besides, it is necessary to educate consumers and make them aware that the proportion of each probiotic strain or their lysates in a package varies from batch to batch. Different combinations of probiotics are more likely to mitigate the leaky gut problems and provide the gut flora with the required needs. Most commercial yogurts or probiotic drugs contain 1 - 9 registered probiotic strains. Recently, it has been demonstrated that formulations of different lactobacilli in association with bifidobacteria were not opposed to each other and had an immunomodulatory effect on Staphylococcus infections [22]. In another study, different probiotic cultures of L. casei, B. longum, B. bifidum or L. casei, L. acidophilus, B. bifi$d u m$, and $B$. longum were found to be on components of future probiotic drugs effective in treating staphylococcosis and for immunity correction [23]. As there seem to have been so many claims on probiotics, the European regulatory legislation requires extensive validation for biological activity of both independent probiotic cultures and their combinations, which would allow for the development of functional probiotic drugs based on monocultures of bifidobacteria and/or lactobacilli or in their various combinations [23].

To individuals that are already healthy, both probiotics and paraprobiotics are still needed to maintain the health status because digestive health, and a balanced gut flora work together in a synergistic way. To that effect, reference [24] prescribed minimum probiotic bacteria intake of $1.5 \times 10^{10}-2.0 \times 10^{10} \mathrm{CFU} /$ day for adults. It is further recommended that children require specific formulations of probiotics with slightly smaller amounts. Likewise, unique formulations of PCFs are required, depending on the targeted beneficial activities in different body organs. Additional epidemiological studies are worth the effort on these subjects as everyone is different and has a distinctive gut flora.

\section{Surface Layer (S-Layer) Proteins in Alleviating Intestinal Disorders and Food Allergy}

S-layer proteins can be isolated and be part of PCFs or paraprobiotics. They are observed to adhere to mucus or intestinal epithelial cells in a competitive nature against enteropathogenic bacteria of Clostridium perfringens [25], Escherichia coli O157:H7, Salmonella Typhimurium [26], and Clostridium difficile, Shigella sp., and Salmonella sp. [27]. Seemingly, the location of S-layer proteins at the outermost surface of some lactobacilli strains implies that it plays a role in adhesion. If the S-layer proteins of lactobacilli are removed or damaged, the adhesion of these lactobacilli to the host epithelial cells is reduced [28]. The S-layer proteins seem to be a perfect surface structure in most of lactobacilli and various bifidobacteria [27]. These 2 genera are also preferred for study due to their wealth in early fecal samples from newborns and their postulated role in common allergies 
improvement. Lactobacilli in particular, enjoy some additional properties that make them suitable for many applications, namely, their generally regarded as safe (GRAS) status, ability to induce mucosal and systemic immune responses against associated antigens, and impressive immunomodulating properties.

S-layer-coated liposomes have been proposed to be excellent candidates to be used as antigen carriers due to their potential to surface-display proteins and epitopes, as well as their intrinsic adjuvant means [29]. Reference [30] have demonstrated the potential to surface-expose epitopes as part of the L. acidophilus S-layer proteins. Single $\mathrm{S}$-protein or $\mathrm{S}_{\mathrm{A}}$-protein was found in more than $10^{5}$ copies per cell, and the emergence of antigens on the surface of lactobacilli was proposed to be useful in the development of oral and/or nasal vaccines [30]. Another approach is that recombinant fusion of allergens and S-layer proteins could improve vaccines for immunotherapy of atopic allergies [31]. The impact made here is that several lactobacilli and bifidobacteria possess additional properties that allow them to halt the spread of common allergies. The S-layer proteins are also found to have aggregation ability of biofilm formation [32]. This is another most significant distinguishing characteristic for scientists to explore the possibilities of probiotic biofilm (p-biofilm) formation in food allergy conditions while restoring a leaky gut caused by either aggressive gut flora or other sources of initiating the inflammations. S-Layer proteins are known to fulfill a wide range of functions such as acting as: 1) structure involved in cell adhesion and surface recognition; 2) protective coats, molecular sieves, and molecule and ion traps; 3) virulence factor in pathogenic organisms; and 4) patterning elements [33].

\section{Gluten, Gliadin, and Glutenin as a Reason for Protein Allergy}

In food chemistry, gluten, gliadin, and glutenin in wheat are classified as conjugated plant protein, prolamins, and glutelins, in that order. It is surprising that most of the individual prolamins fall into the variety of conjugated plant proteins. The prolamin content of gluten is usually taken as $50 \%$. Gluten is a tenacious elastic protein, consisting of a mixture of glutenin and gliadin, especially of wheat flour that gives cohesiveness to dough. Glutenin proteins are similarly elastomeric, form insoluble polymer networks with rubber-like elasticity, and build multidomain cross-linkers too [34]. Intuitively, glutenin is also challenging because it cannot only be recognized by the same intestinal gliadin-specific $\mathrm{T}$ cells among other $\mathrm{T}$-cell populations, but also demonstrates that the glutenin-specific $\mathrm{T}$ cells most likely are an integral part of the pathological T-cell repertoire within the celiac lesions [35]. The ingestion of these gluten proteins from wheat, rye, barley, or their crossbred varieties induces an inflammatory response that destroys the villous structure of the intestine and impairs its ability to absorb nutrients, especially in patients suffering from celiac disease [36] [37]. Until today, finding a replacement of gluten proteins in bakery products remains a significant technological challenge. The examples of prolamin from wheat is gliadin, from barley is hordein, from rye is secalin, and from oats is avenin. It is reported that gluten intolerance is a physiological disorder that leads to gliadin allergy, from which more than 500,000 people suffer in the United States alone [37].

Although not all gluten varieties seem to have the same effect in people with celiac disease or non-celiac gluten sensitivity, they can all be problematic for digestive health [6]. Foxtail millet, pearl millet (Omahangu), and sorghum are gluten-free and have a legitimate chance of being commercialized for the food-based control of gluten problem. Rice, rye, corn, amaranth, teff, quinoa, buckwheat, and chia are also identified to be gluten-free. For individuals suffering from gluten intolerance and/or celiac disease, grains containing gluten with the combination of some amino acids in the gliadin family seem to create the most problems. The grains blacklisted on a gluten-free diet include wheat and its relatives (triticale, spelt, kamut), barley, rye, and sometimes oats.

Oats do not contain gluten per se, but they are almost always contaminated with gluten (unless specifically labeled gluten-free) since they are usually processed on the same equipment used for wheat and other gluten-containing grains [6]. Table 1 shows different kinds of processed foods where gluten can hide in a diet [6]. However, the extent to whether the leaky gut formation is only in gluten allergy conditions or any allergen proteins from different food create a leaky gut system needs to be further investigated.

\section{Concluding Remarks}

It is challenging to predict that the food supply can be made allergen-free because most food proteins and even sulfites (in foods) are potential food allergens. Various diets that allergic people love best are probably not always the best for their precarious conditions if eaten moderately or in excessive amounts. Supplementation of probiotic formulations (live probiotic cells, paraprobiotics, and/or probiotic-paraprobiotics in combinations) ap- 
Table 1. Foods where gluten, gliadin, and glutenin hide in a diet.

\begin{tabular}{|c|c|}
\hline Diet & Food products where gluten, gliadin, and glutenin hide \\
\hline Breakfast & $\begin{array}{l}\text { Breakfast cereals, muesli, regular oatmeal, bagels, toasts, muffins, croissants, pancakes, } \\
\text { smoothies made with wheat germ, and waffles. }\end{array}$ \\
\hline Lunch & $\begin{array}{l}\text { Burgers (maybe in the bread and the meat patty), Sandwiches (perhaps in the deli meat, pizzas, and bread), breaded chicken, } \\
\text { imitation bacon, salads with croutons, barley soup, dumplings, sushi (in the tempura, soy sauce, or imitation crab), soups (in } \\
\text { the noodles or the thickening agent), bulgur salad and seitan. }\end{array}$ \\
\hline Dinner & $\begin{array}{l}\text { Lasagna, spaghetti, rice and risotto (particularly from the seasonings), meat pies (from the sauce in the filling and the crust), } \\
\text { noodles, stuffing, chicken nuggets, sausages, bulgur, frozen French fries (from the coating), and couscous. }\end{array}$ \\
\hline Snacks & Crackers, granola bars, nachos, yogurt with granola, potato chips (from the flavoring), and pretzels. \\
\hline Desserts & $\begin{array}{l}\text { Cakes, cookies, pies, graham crackers, muffins, scones, puddings, baked goods } \\
\text { (made from wheat flour or graham flour, or containing glutenous ingredients), brown rice syrup, candies, and some yogurts. }\end{array}$ \\
\hline Seasonings & $\begin{array}{l}\text { Some tamari sauces, soy sauce, malt vinegar, some salad dressing, many seasonings, sauces and marinades, } \\
\text { flavorings, gravies, spice blends, and more. }\end{array}$ \\
\hline Alcohol & Spirits made from grains, beer, various mixes and liqueurs (from the thickening agents) \\
\hline Others & $\begin{array}{l}\text { Some vitamin and mineral supplements, play dough, lipsticks, some medications, } \\
\text { personal hygiene and cosmetic products, communion wafers, glue on envelopes and stamps, and many others. }\end{array}$ \\
\hline
\end{tabular}

pears to have both an immediate and long-term successes in curbing common allergies, depending on the probiotic containing product taken. Thus, screening of anti-allergy probiotic strains would be a leading step in research about formulating PCFs for common allergies. As food-allergy has been observed to be intuitively outgrown with age, this expresses the maturity and advancement of the digestion system in human. However, extensive cooking of the plant source allergens appears to simplify the protein quality and palatability of the celiac diet. Extremely dry heating by modern food-processing devices including irradiation, ionization, and a few others need to be investigated further because they are alleged to create complex and diverse group of compounds, called advanced glycation end-products (AGEs), and/or advanced lipid oxidation end-products (ALEs). Once these products are partially absorbed into the systemic circulation, they also may have adverse health effects in human. The answer now appears to depend on the adequate intake of probiotics or paraprobiotics in their different formulations.

\section{Acknowledgements}

Special Grant (2013M541397) from China Postdoctoral Science Foundation is acknowledged for funding. To John Justo Ambuchi for proofreading.

\section{References}

[1] FAO/WHO (2002) Guidelines for the Evaluation of Probiotics in Food. http://www.who.int/entity/foodsafety/publications/ss management/probiotics2/en

[2] Taverniti, V. and Guglielmetti, S. (2011) The Immunomodulatory Properties of Probiotic Microorganisms Beyond their Viability (Ghost Probiotics: Proposal of Paraprobiotic Concept). Genes and Nutrition, 6, 261-274. http://dx.doi.org/10.1007/s12263-011-0218-x

[3] Finamore, A., Roselli, M., Britti, M.S., Merendino, N. and Mengheri, E. (2012) Lactobacillus rhamnosus GG and Bifidobacterium animalis MB5 Induce Intestinal But Not Systemic Antigen-Specific Hyporesponsiveness in Ovalbumin-Immunized Rats. The Journal of Nutrition, 142, 375-381. http://dx.doi.org/10.3945/jn.111.148924

[4] Huang, H.W., Hsu, C.P., Yang, B.B. and Wang, C.Y. (2014) Potential Utility of High-Pressure Processing to Address the Risk of Food Allergen Concerns. Comprehensive Reviews in Food Science and Food Safety, 13, 78-90. http://dx.doi.org/10.1111/1541-4337.12045

[5] Ouwehand, A.C. (2007) Antiallergic Effects of Probiotics. The Journal of Nutrition, 137, 794S-797S.

[6] Jacob, A. (2013) Digestive Health with REAL Food: A Practical Guide to an Anti-inflammatory, Nutrient Dense Diet for IBS and Other Digestive Issues. Paleo Media Group, Bend.

[7] Sicherer, S.H. (2013) Food Allergies: A Complete Guide for Eating When Your Life Depends On It. The Johns Hopkins University, Baltimore. http://dx.doi.org/10.1201/b15358 
[8] Holt, P.G. (1998) Mucosal Immunity in Relation to the Development of Oral Tolerance/Sensitization. Allergy, 53, 16-19. http://dx.doi.org/10.1111/j.1398-9995.1998.tb04952.x

[9] Cross, M.L., Stevenson, L.M. and Gill, H.S. (2001) Anti-Allergy Properties of Fermented Foods: An Important Immunoregulatory Mechanism of Lactic Acid Bacteria? International Immunopharmacology, 1, 891-901. http://dx.doi.org/10.1016/S1567-5769(01)00025-X

[10] Kidd, P. (2003) Th1/Th2 Balance: The Hypothesis, Its Limitations, and Implications for Health and Disease. Alternative Medicine Review, 8, 223-246.

[11] He, F., Ouwehand, A.C., Isolauri, E., Hashimoto, H., Benno, Y. and Salminen, S. (2001) Comparison of Mucosal Adhesion and Species Identification of Bifidobacteria Isolated from Healthy and Allergic Infants. FEMS Immunology and Medical Microbiology, 30, 43-47. http://dx.doi.org/10.1111/j.1574-695X.2001.tb01548.X

[12] Isolauri, E., Sütas, Y., Kankaanpää, P., Arvilommi, H. and Salminen, S. (2001) Probiotics: Effects on Immunity. The American Journal of Clinical Nutrition, 73, 444s-450s.

[13] Pelto, L., Isolauri, E., Lilius, E.M., Nuutila, J. and Salminen, S. (1998) Probiotic Bacteria Down-Regulate the Milk-induced Inflammatory Response in Milk-Hypersensitive Subjects But Have an Immunostimulatory Effect in Healthy Subjects. Clinical and Experimental Allergy, 28, 1474-1479.

http://dx.doi.org/10.1046/j.1365-2222.1998.00449.x

[14] Sanders, M.E., Morelli, L. and Tompkins, T.A. (2003) Sporeformers as Human Probiotics: Bacillus, Sporolactobacillus, and Brevibacillus. Comprehensive Reviews in Food Science and Food Safety, 2, 101-110. http://dx.doi.org/10.1111/j.1541-4337.2003.tb00017.x

[15] Hosoi, T., Ametani, A., Kiuchi, K. and Kaminogawa, S. (2000) Improved Growth and Viability of Lactobacilli in the Presence of Bacillus Subtilis (Natto), Catalase, or Subtilisin. Canadian Journal of Microbiology, 46, 892-897. http://dx.doi.org/10.1139/w00-070

[16] Taylor, A.L., Dunstan, J.A. and Prescott, S.L. (2007) Probiotic Supplementation for the First 6 Months of Life Fails to Reduce the Risk of Atopic Dermatitis and Increases the Risk of Allergen Sensitization in High-Risk Children: A Randomized Controlled Trial. Journal of Allergy and Clinical Immunology, 119, 184-191. http://dx.doi.org/10.1016/j.jaci.2006.08.036

[17] Del-Immune, V. (2014) Del-Immune V ${ }^{\circledR}$. http://www.delimmune.com/research

[18] Bioray (2014) CytoFlora ${ }^{\mathrm{TM}}$. http://www.bioray.com/cytoflora

[19] Wang, S.-M., Zhang, L.-W., Fan, R.-B., et al. (2014) Induction of HT-29 Cells Apoptosis by Lactobacilli Isolated from Fermented Products. Research in Microbiology, 165, 202-214. http://dx.doi.org/10.1016/j.resmic.2014.02.004

[20] West, R.D., Roberts, E., Sichel, L.S. and Sichel, J. (2013) Improvements in Gastrointestinal Symptoms among Children with Autism Spectrum Disorder Receiving the Delpro® Probiotic and Immunomodulator Formulation. Journal of Probiotics and Health, 1, 1.

[21] Shigwedha, N. and Jia, L. (2013) Bifidobacterium in Human GI Tract: Screening, Isolation, Survival and Growth Kinetics in Simulated Gastrointestinal Conditions. In: Kongo, M., Ed., Lactic Acid Bacteria-R \& D for Food, Health and Livestock Purposes, InTech, Croatia, 281-308. http://dx.doi.org/10.5772/50457

[22] Starovoitova, S., Kishko, K., Lazarenko, L., Shynkarenko, L., Spivak, M. and Nikolaychuk, M. (2010) Cholesterase Activity of New Lacto- and Bifidobacteria Strains In-vitro. Scientific Bulletin of Uzhhorod University, 27, 42-45 (in Russian).

[23] Lazarenko, L., Babenko, L. and Sichel, L.S. (2012) Antagonistic Action of Lactobacilli and Bifidobacteria in Relation to Staphylococcus Aureus and Their Influence on the Immune Response in Cases of Intravaginal Staphylococcosis in Mice. Probiotics and Antimicrobial Proteins, 4, 78-89. http://dx.doi.org/10.1007/s12602-012-9093-z

[24] Campbell-McBride, N. (2010) Gut and Psychology Syndrome: Natural Treatment for Autism, Dyspraxia, A.D.D., Dyslexia, A.D.H.D., Depression, Schizophrenia. Medinform Publishing Cambridge.

[25] Matsumoto, M., Tani, H., Ono, H., Ohishi, H. and Benno, Y. (2002) Adhesive Property of Bifidobacterium Lactis LKM512 and Predominant Bacteria of Intestinal Microflora to Human Intestinal Mucin. Current Microbiology, 44, 212-215. http://dx.doi.org/10.1007/s00284-001-0087-4

[26] Chen, X.Y., Xu, J.J., Shuai, J.B., Chen, J.S., Zhang, Z.F. and Fang, W.H. (2007) The S-Layer Proteins of Lactobacillus Crispatus Strain ZJ001 Is Responsible for Competitive Exclusion against Escherichia Coli O157:H7 and Salmonella Typhimurium. International Journal of Food Microbiology, 115, 307-312. http://dx.doi.org/10.1016/j.ijfoodmicro.2006.11.007

[27] Xue, C., Zhang, L., Li, H., et al. (2013) Functionality of the S-Layer Proteins from Lactobacillus in the Competitive Against Enteropathogens Infection. European Food Research and Technology, 236, 249-255. http://dx.doi.org/10.1007/s00217-012-1871-z 
[28] Lee, Y.K., Puong, K.Y., Ouwehand, A.C. and Salminen, S. (2003) Displacement of Bacterial Pathogens from Mucus and Caco-2 Cell Surface by Lactobacilli. Journal of Medical Microbiology, 52, 925-930. http://dx.doi.org/10.1099/jmm.0.05009-0

[29] Hollmann, A., Delfederico, L., Glikmann, G., De Antoni, G., Semorile, L. and Disalvo, E.A. (2007) Characterization of Liposomes Coated with S-Layer Proteins from Lactobacilli. Biochimica et Biophysica Acta (BBA)-Biomembranes, 1768, 393-400. http://dx.doi.org/10.1016/j.bbamem.2006.09.009

[30] Smit, E., Jager, D., Martinez, B., Tielen, F.J. and Pouwels, P.H. (2002) Structural and Functional Analysis of the S-Layer Protein Crystallisation Domain of Lactobacillus Acidophilus ATCC 4356: Evidence for Protein-Protein Interaction of Two Subdomains. Journal of Molecular Biology, 324, 953-964. http://dx.doi.org/10.1016/S0022-2836(02)01135-X

[31] Gerstmayr, M., Ilk, N., Schabussova, I., et al. (2007) A Novel Approach to Specific Allergy Treatment: The Recombinant Allergen-S-Layer Fusion Protein Rsbsc-Bet V 1 Matures Dendritic Cells that Prime Th0/Th1 and IL-10-Producing Regulatory T Cells. The Journal of Immunology, 179, 7270-7275.

[32] Pérez, P.F., Minnaard, Y., Disalvo, E.A. and De Antoni, G.L. (1998) Surface Properties of Bifidobacterial Strains of Human Origin. Applied and Environmental Microbiology, 64, 21-26.

[33] Egelseer, E.M., Ilk, N., Pum, D., et al. (2009) S-Layers, Microbial, Biotechnological Applications. Encyclopedia of Industrial Biotechnology: Bioprocess, Bioseparation, and Cell Technology. John Wiley \& Sons Ltd., Chichester.

[34] Shewry, P.R., Halford, N.G., Belton, P.S. and Tatham, A.S. (2002) The Structure and Properties of Gluten: An Elastic Protein from Wheat Grain. Philosophical Transactions of the Royal Society of London. Series B: Biological Sciences, 357, 133-142. http://dx.doi.org/10.1098/rstb.2001.1024

[35] Molberg, Ø., Solheim, F.N., Jensen, T., et al. (2003) Intestinal T-Cell Responses to High-Molecular-Weight Glutenins in Celiac Disease. Gastroenterology, 125, 337-344. http://dx.doi.org/10.1016/S0016-5085(03)00890-4

[36] Farrell, R.J. and Kelly, C.P. (2002) Celiac Sprue. New England Journal of Medicine, 346, 180-188. http://dx.doi.org/10.1056/NEJMra010852

[37] Rai, K.N., Gowda, C.L.L., Reddy, B.V.S. and Sehgal, S. (2008) Adaptation and Potential Uses of Sorghum and Pearl Millet in Alternative and Health Foods. Comprehensive Reviews in Food Science and Food Safety, 7, 340-352. 